\title{
The role of experience without set in figural after-effects'
}

\author{
P. C. Dodwell and Lorna Gaze \\ QUEEN'S UNIVERSITY
}

\begin{abstract}
Abstraet
It has been shown that reports of figural after-effects (FAEs) are very susceptible to the effects of an inducing set in otherwise experimentally naive Ss. Such Ss do not generally report FAEs in the absence of an induced set. This may be so either because FAEs are artifacts of set and not real perceptual after-effects, or because naive Ss lack practice in making fine visual discriminations and hence do not observe the small after-effects present in a display. It is demonstrated that in at least one situation the latter alternative is the more probable.

\section{Problem}

It was shown in the preceding paper (Gaze \& Dodwell, 1965) that large pseudo-FAEs, both positive and negative, can be obtained from otherwise naive Ss under an appropriate inducing set, and that without specific set such Ss do not report reliable FAEs. The latter finding has also been reported by several other investigators. Before concluding that FAEs may be simpleartifacts of a set induced by specific instructions or the general expectations of persons familiar with the classical work on FAE, another alternative should be explored. It is well known that generally some increase in the precision of psychophysical and other similar judgments occurs during early practice, even without specific knowledge of results. One might therefore enquire whether the negative FAE results with naive Ss are due simply to lack of practice at making precise judgments about such things as lengths of lines and distance between contours.

The first experiment was undertaken to investigate whether or not experimentally naive Ss with no knowledge of FAE, and no induced set, would observe FAEs after pre-training on tasks involving judgment of the equality of the lengths of two lines. The pre-training task was quite different from the FAE judgment task, so that no specific transfer from one to the other should be present.

The second experiment investigated the effect of pretraining with the actual stimuli to be used in obtaining FAE reports. During pre-training Ss had to make precisely the same sorts of judgment as in the FAE situation, namely to judge the orientations of straight lines. Köhler \& Wallach (1944) reported that inspection of a line in one orientation in the inspection figure could alter the perception of similarly oriented lines in the test figure. Similar findings have been reported by others, but these reports were generally not obtained under set-free conditions, so are open to the objections made earlier (Gaze \& Dodwell, 1965). It is known that the precision with which line orientations can be judged is a function of the orientations, being greatest for lines near the horizontal and vertical, and less at positions midway between these orientations (e.g., Jastrow, 1893). It was therefore predicted that FAEs of this sort would be most readily obtained near the horizontal and vertical positions, and with more difficulty (even after practice) in other positions, if indeed practice and precision of judgments are relevant factors (see experiment 1).

\section{Experiment 1}

\section{Method}

The Ss were 40 experimentally naive summer school students, who knew nothing about FAEs. The FAE apparatus was a simplified version of that described in the preceding paper (Gaze \& Dodwell, 1965). The test and inspection figures were virtually identical with those shown in Fig. 1 of that paper, but were painted as black lines on a matte white board. The moveable bar of the test figure was on a sliding panel fitted into a groove on the test figure board, which could be adjusted by $\mathrm{E}$. The pre-training apparatus consisted of a standard Lafayette visual illusion set (Muller-Lyer \& Horizontal-Vertical Illusions, white figures on a black background). The experiment was designed as a $2 \times 2 \times 2$ factorial with two pre-training tasks (MüllerLyer \& Horizontal-Vertical), two levels of pre-training (10 and 50 trials) and two levels of knowledge of results (knowledge and no knowledge) on pre-training trials.

Ss were assigned randomly to the eight groups, and in the pretraining task free inspection of the appropriate figure was allowed, E adjusting the illusion until the comparison line appeared to be equal in length to the standard line in the figure. In the knowledge groups the comparison line was set to true equality after each judgment by $S$, and then moved well beyond the position of apparent equality (i.e., made longer). In the no knowledge groups the second step was taken immediately $\mathrm{S}$ had completed his judgment.

After pre-training the FAE trials began at once, $S$ being given five practice trials, 10 control trials, 10 experimental trials and a further 10 control trials. Timing and calculation of FAE were the same is in the previous experiment (Gaze \& Dodwell, 1965). Other conditions were similar (headrest, monocular fixation) except that in this case $E$ adjusted the moveable panel of the test figure until $\mathrm{S}$ said "stop." No readjustment was allowed.

Table 1. Mean FAE scores, all conditions

\begin{tabular}{lrccc} 
& \multicolumn{2}{c}{ H-V } & \multicolumn{2}{c}{ M-L } \\
& $\mathrm{K}$ & $\mathrm{NK}$ & $\mathrm{K}$ & $\mathrm{NK}$ \\
10 practice & $-16.0^{\prime}$ & $-1.5^{\prime}$ & $-15^{\prime}$ & $-39^{\prime}$ \\
50 practice & $45.5^{\prime}$ & $49.5^{\prime}$ & $56.5^{\prime}$ & $96^{\prime}$
\end{tabular}

All measurements are in minutes of arc. Only the overall practice effect was reliable.

\section{Results}

The mean FAEs for the eight different conditions are shown in Table 1. Analysis of variance showed that the only significant main effect was practice $(F=6.37$; df $(1,32) ; p<.025)$. The other $F$ ratios, both for main effects and interactions, were close to or less than 1. Inspection of the means in Table 1 demonstrates clearly that 50 trials of practice at judging line lengths resulted 
Table 2

Design for Experiment 2

Gp I

a 60 practice trials

60 practice trials
Day 2

$0^{\circ} \& 70^{\circ}$

$20^{\circ} \& 90^{\circ}$

FAE trials

FAE trials

$0^{\circ} \& 70^{\circ}$

$20^{\circ} \& 90^{\circ}$

Gp II

a no practice

b no practice

FAE trials

FAE trials

$0^{\circ} \& 70^{\circ}$

$20^{\circ} \& 90^{\circ}$

There were no significant effects for practice or orientation. The FAE for $90^{\circ}$ only (groups Ib \& II b combined) was significantly different from zero (overall mean FAE at $90^{\circ}=1^{\circ}$ exactly).

in larger FAEs than 10 trials. Since all the other sources of variance are negligible, a $t$ test was performed on the overall mean for the 50 trial groups, showing that this was significantly different from zero $(t=3.2 ; p<.01)$. It is worth noting that all the 10 -trial groups showed negative effects (the settings after inspection were smaller than the control settings) although their overall mean was not significantly different from zero.

\section{Method}

\section{Experiment 2}

The Ss were 24 experimentally naive summer school students, who knew nothing about FAEs. The apparatus consisted of two parts, an I figure, and a $\mathrm{T}$ figure for testing FAEs which was also used for pretraining in judging orientations. The I figure was a plain white board with a black circular fixation mark at its center, $.5 \mathrm{~cm}$ in diameter. Forty eight $\mathrm{mm}$ to the right of it and centered $10 \mathrm{~mm}$ above the horizontal bisecting the board, was a rotatable straight black line, $1 \mathrm{~mm}$ thick and $93 \mathrm{~mm}$ long. The test figure was a similar board, but with a second rotatable line of the same dimensions attached $48 \mathrm{~mm}$ to the left of the fixation point. A headrest and right monocular fixation from a distance of $70 \mathrm{~cm}$ were used throughout. The Ss were divided into four groups, as shown in Table 2. Since experiment 1 indicated that neither type of pre-training figure nor knowledge of results were of importance insofar as subsequent FAE measurements are concerned, pre-training Ss were all run on the same figure (but with different orientations) and without knowledge of results. In a pre-training trial, one of the lines in the $\mathrm{T}$ figure (the comparison line) was set at the appropriate orientation and the other was set $20^{\circ}$ away from this orientation (clockwise or anticlockwise). E then rotated the second line towards the orientation of the first, until S said "stop." The task was to set the two lines in parallel, and no readjustment was allowed. In an FAE trial the I figure was inspected in a particular orientation, either on the left or the right of the fixation point. Immediately afterwards the $\mathrm{T}$ figure was viewed, with the line on the opposite side of the fixation point in the same orientation as the line of the I figure. The line on the same side as the I figure line was set $20^{\circ}$ off this orientation, and rotated towards it as before. The two orientations of practice and/or FAE stimuli, side of the I and/or comparison line, clockwise/anticlockwise setting and rotation were randomized independently for each $\mathrm{S}$, under the restriction that each condition within a pair occurred equally often over the 60 trials of pre-training and/or over the control and experimental FAE trials. In FAE trials all Ss were given four practice trials, followed by 12 control, 12 experimental and again 12 control trials. The inspection time was $60 \mathrm{sec}$, with $120 \mathrm{sec}$. of rest between experimental trials. FAEs were calculated as the difference between the mean control and experimental setting for each $\mathrm{S}$ at each comparison position. The direction from which the test line approached the orientation of the comparison line was ignored in this calculation, so that a control setting closer to the comparison orientation than the experimental setting always gave a positive difference (FAE score).

\section{Results}

In this experiment practice at judging line orientation had no detectable effect on subsequent FAE measurements. Analysis of variance on the FAE scores showed that differences between FAE scores for different orientations were not reliable. However, the FAE score for the $90^{\circ}$ (horizontal) orientation was reliably different from zero when the practice and no-practice groups were combined, $(t=3.7 ;$ df $11 ; p<.01)$. None of the other FAE scores was significantly greater than zero.

\section{Discussion}

The results of these two experiments are consistent with the hypothesis that, at least in some situations, the failure of naive Ss to report FAEs is due to their lack of practice in making fine visual judgments. It is now established beyond reasonable doubt that FAEs are genuine perceptual phenomena. However, when measured under set-free conditions they appear to be smaller than has sometimes been claimed, and Ss may need practice at the appropriate sort of judgment before seeing them. In the first experiment a positive practice effect was demonstrated, but not in the second. In experiment 2 the failure of practice to lead to an improvement in FAE detection could be due to any one of three differences from experiment 1 , or a combination of them. First, a different type of judgment was involved (orientation rather than distance), and conceivably practice takes longer to show an effect here. Secondly, the same stimulus display was used for pretraining and FAE detection; it is difficult to see why this might lead to failure of a practice effect to show up. Thirdly, there is the fact that in the first experiment judgments were made about visual illusions during pretraining, but not in the second. Decisions between these alternatives would require further experiments.

In experiment 2 a positive FAE was shown for the $90^{\circ}$ (horizontal) line orientation but not for other orientations. This is also consistent with the hypothesis, if it be granted that horizontal and vertical judgments tend to be much more highly practiced, even in experimentally naive Ss, than others. Although the mean vertical FAE score was not reliably different from zero, it came close to being so.

The displays used for measuring FAEs in experiment 2 were different from those used conventionally for measurement of orientation FAEs. There were two reasons for this. In the first place, if FAEs are due to fatigue or adaptation in cortical neurons for simple receptive fields (Hubel \& Wiesel, 1962) the display used should be optimal, since the test line is rotated about its center, which is in the same position as was the inspection line center. In the second place, the display used was simple to construct and operate, and allowed a straightforward quantitative computation of the FAE to be made. In most previous displays orientation and position changes with FAE have been confounded.

\section{References}

GAZE, LORNA, \& DODWELL, P.C. The role of induced set in figural after-effects. Psychon. Sci., 1965, 2, 275-276.

HUBEL, D. H., \& WIESEL, T. N. Receptive fields, binocular interaction and functional architecture in the cat's visual cortex. J. Physiol., 1962, 160, 106-154.

JASTROW, J。 On the judgment of horizontal, vertical and oblique positions of lines. Amer. J. Psychol., 1893, 5, 220-223.

KOHLER, W., \& WALLACH, H. Figural after-effects. Proc. Amer. Phil. Soc., 1944, 88, 269-357.

\section{Note}

1. This research was supported by grants to the first author from the National Research Council of Canada (APA 44) and from the Arts Research Committee of Queen's. 